\title{
RADIAÇÃO GAMA NA CONSERVAÇÃO DO SUCO NATURAL DE LARANJA
}

\author{
Juliana lemma'; André Ricardo Alcarde ${ }^{1,3 *}$; Rachel Elisabeth Domarco ${ }^{2}$; Marta Helena Fillet \\ Spoto²; Lucimara Blumer'; Clarice Matraia² \\ ${ }^{1}$ Pós-Graduandos do Laboratório de Irradiação de Alimentos e Radioentomologia - CENA/USP. \\ ${ }^{2}$ Laboratório de Irradiação de Alimentos e Radioentomologia - CENA/USP, C.P. 96 - CEP: 13400-970 - Piracicaba, SP. \\ ${ }^{3}$ Bolsista da FAPESP. \\ *e-mail: aalcarde@cena.usp.br
}

RESUMO: Este trabalho estudou o efeito da radiação gama na população microbiológica, teor de sólidos solúveis, acidez titulável, $\mathrm{pH}$ and conteúdo de vitamina $\mathrm{C}$ do suco natural de laranja. A atividade microbiana pode causar a deterioração do suco de laranja. A irradiação é um processo de conservação de alimentos através da eliminação dos microrganismos, porém pode afetar algumas características do produto. $O$ delineamento experimental foi em esquema fatorial $4 \times 5$ referentes ao controle mais 3 doses de radiação $(2,0 ; 4,0$ and $6.0 \mathrm{kGy})$ e 5 períodos de armazenamento $(1,7,14,21$ e 28 dias) com 2 repetições. As amostras de suco natural foram extraídas de laranjas da variedade "Pêra", irradiadas a uma taxa de dose de 2,0 kGy por hora (cobalto-60), e em seguida foram armazenadas sob refrigeração $\left(5 \pm 3^{\circ} \mathrm{C}\right)$. Os resultados mostraram pequenas variações no teor de sólidos solúveis, acidez titulável e $\mathrm{pH}$, em relação aos tratamentos testados. A razão sólidos solúveis/acidez titulável foi determinada e também mostrou pequena variação em todos os tratamentos. Com o aumento da dose de radiação e o prolongamento do período de armazenamento houve redução no conteúdo de vitamina $\mathrm{C}$ do suco. A radiação gama se mostrou eficaz na redução da população microbiana do suco de laranja.

Palavras-chave: radiação gama, suco de laranja, conservação

\section{GAMMA RADIATION AND THE CONSERVATION OF NATURAL ORANGE JUICE}

ABSTRACT: The effect of gamma radiation was evaluated on the microbiological population, soluble solids content, acidity, $\mathrm{pH}$ and ascorbic acid content of natural orange juice. Microbial activity may cause deterioration of orange juice. Irradiation is a process of food conservation which eliminates microorganisms. Neverthless radiation may affect some characterisitics of irradiated foods. The experimental design was a 4x5 factorial scheme, including control and 3 rates of irradiation (2.0, 4.0 and $6.0 \mathrm{kGy}$ ) and 5 storage periods $(1,7,14,21$ and 28 days), with 2 replicates. Samples of juice were extracted from variety "Pera" oranges and irradiated at a rate of $2.0 \mathrm{kGy} / \mathrm{h}$ ( ${ }^{60} \mathrm{Cobalt}$ ) and thereafter stored at $5 \pm 3^{\circ} \mathrm{C}$. Results showed small changes in soluble solids content, acidity and $\mathrm{pH}$, for all treatments. The ratio soluble solids/acidity was also determined and showed little variation for all treatments. There was a reduction on ascorbic acid content of the orange juice with increased radiation dosage and storage time. Gamma radiation was effective in reducing the microbiological population of the juice.

Key words: gamma radiation, orange juice, conservation

\section{INTRODUÇÃO}

Devido a aspectos técnicos e econômicos, um dos principais problemas enfrentados pelas empresas produtoras de suco de laranja é o seu congelamento à temperatura de $18^{\circ} \mathrm{C}$ negativos, necessário para a conservação e distribuição do produto. Em vista disto, torna-se interessante o estudo de um processo alternativo para a conservação deste suco.
O tratamento de frutas e vegetais com radiações ionizantes tem como principal finalidade assegurar sua preservação, isto é, aumentar o tempo de conservação do alimento. Este processo pode envolver a inativação de microrganismos (principalmente fungos, bactérias e leveduras), o retardo da maturação e a desinfestação, dentre outros mecanismos.

Assim como outras técnicas de processamento de alimentos, a irradiação pode causar mudanças na composição química e no 
valor nutritivo. A natureza e extensão destas mudanças dependem essencialmente do tipo, variedade e composição do alimento, da dose de radiação recebida e das condições ambientais durante e após a irradiação (Wiendl, 1984).

Estudos demonstram que, dentre as vitaminas hidrossolúveis, a $C$ é a mais sensível à radiação (Josephson, 1978). No entanto, convém lembrar que, por ser uma vitamina relativamente lábil, resultados semelhantes de sua destruição ocorrem nos tratamentos de alimentos pelo uso do calor (Wiendl, 1984).

Sendo a inativação de microrganismos um ponto chave na preservação de alimentos, uma pesquisa realizada por Domarco et al. (1996) mostrou que doses de radiação de 1,0;2,5 e 5,0 kGy foram efetivas em controlar a proliferação da levedura Saccharomyces cerevisiae em suco de laranja por 90 dias de armazenamento a 0,5 e $25^{\circ} \mathrm{C}$.

Segundo Spoto (1988), um tratamento promissor para frutas, suco de frutas e vegetais é a combinação da radiação com a refrigeração, pois a esterilização com a irradiação poderia requerer uma dose elevada e ainda assim não promover a inativação de enzimas, responsáveis pelas mudanças sensoriais durante 0 armazenamento.

O objetivo do presente trabalho foi verificar o efeito da radiação gama sobre o teor de sólidos solúveis, acidez titulável, razão sólidos solúveis/acidez titulável, pH, conteúdo de vitamina $\mathrm{C}$ e população microbiana do suco natural de laranja, visando sua conservação.

\section{MATERIAL E MÉTODOS}

As amostras de suco foram obtidas de laranjas da variedade "Pêra". Após a lavagem das laranjas, foi realizada a extração do suco em espremedor manual; em seguida, o suco foi acondicionado em frascos de vidro âmbar de 120 $\mathrm{ml}$, os quais foram colocados no irradiador modelo Gammabeam 650, fabricado pela Nordion Canadian, com atividade de $1,92 \times 10^{14} \mathrm{~Bq}$, a uma taxa de dose de 2,0 kGy/h. As doses de radiação utilizadas foram 0,0 (controle); 2,0; 4,0 e 6,0 kGy. Após a irradiação, o suco foi armazenado sob refrigeração $\left(5 \pm 3^{\circ} \mathrm{C}\right)$ e analisado química e microbiologicamente aos 1, 7, 14, 21 e 28 dias de armazenamento.

O estudo da estabilidade do suco foi realizado através das seguintes determinações fisico-químicas: teor de sólidos solúveis (SS), expressos em ${ }^{\circ}$ Brix a $20^{\circ} \mathrm{C}$, utilizando-se um refratômetro Reichert-Jung Auto Abbe, modelo 10500/10501, Leica Inc., Buffalo-NY, USA; pH, medido em potenciômetro modelo DMPH-2 da DIGIMED-Brasil; acidez titulável (AT), calculada como o volume em mililitros de $0,3125 \mathrm{~N} \mathrm{NaOH}$ requeridos para titular $100 \mathrm{~mL}$ de suco até $\mathrm{pH}$ 8,2, expressa em porcentagem de ácido cítrico; conteúdo de vitamina $\mathrm{C}$, através de método titulométrico baseado na redução do indicador 2,6-diclorobenzenoindofenol pelo ácido ascórbico, sendo os resultados expressos em $\mathrm{mg}$ de vitamina C por $100 \mathrm{~mL}$ de suco (Jacobs, 1958). Também foi calculada a razão sólidos solúveis/ acidez titulável (SS/AT).

$A$ análise microbiológica baseou-se na determinação do número de unidades formadoras de colônia (UFC) dos microrganismos mesofílicos aeróbios presentes por $\mathrm{mL}$ de suco de laranja. Para isto, foi realizada a semeadura em profundidade em meio PCA $(2,5 \%$ extrato de levedura - 5,0\% triptona - 1,0\% dextrose - 1,5\% agar), com incubação a $35^{\circ} \mathrm{C}$ por 72 horas (Swanson et al., 1992).

O delineamento experimental empregado foi o inteiramente casualizado. Os tratamentos envolvidos no experimento foram definidos através do esquema fatorial $4 \times 5$, referente a quatro níveis de dose de radiação $(0,0 ; 2,0 ; 4,0$ e 6,0 kGy) e cinco níveis de período de armazenamento (1, 7, 14, 21 e 28 dias), com duas repetições por tratamento.

Os resultados foram analisados estatisticamente através da análise de variância com aplicação do teste $F$. Quando o teste $F$ foi significativo ( $P \leq 0,05)$, a análise estatística teve continuidade aplicando-se a análise de regressão polinomial até $2^{\circ}$ grau. Todas as análises foram realizadas utilizando-se o programa estatístico SAS (1990) para microcomputador.

\section{RESULTADOS E DISCUSSÃO}

Os valores obtidos através do teste $F$, aplicado à análise de variância, para as características fisico-químicas do suco de laranja, encontram-se na TABELA 1.

O teor de sólidos solúveis foi afetado significativamente, ao nível de $5 \%$, pela interação dose de radiação e período de armazenamento (TABELA 1). Constatou-se uma relação linear significativa entre a dose de radiação e o teor de sólidos solúveis no $1^{\circ}, 14^{\circ}$ e $21^{\circ}$ dia de armazenamento (Figura 1), enquanto que nos demais períodos não se observou nenhuma relação. 
TABELA 1 - Valores do teste F, aplicados à análise de variância, para as características fisico-químicas do suco de laranja.

\begin{tabular}{|c|c|c|c|c|c|c|c|}
\hline \multirow[b]{2}{*}{ C.V. } & \multirow[b]{2}{*}{ G.L. } & \multicolumn{6}{|c|}{ VALOR F } \\
\hline & & $\begin{array}{l}\text { SÓLIDOS } \\
\text { SOLÚVEIS }\end{array}$ & $\begin{array}{l}\text { ACIDEZ } \\
\text { TITULÁVEL }\end{array}$ & SS/AT & $\mathrm{pH}$ & $\begin{array}{c}\text { ÁCIDO } \\
\text { ASCÓRBICO }\end{array}$ & $\mathrm{UFC} / \mathrm{mL}$ \\
\hline DOSE (D) & 3 & $8,90^{*}$ & $10,46^{*}$ & $19,08^{*}$ & $50,52^{*}$ & $134,19^{*}$ & $94,70^{*}$ \\
\hline PERÍODO (P) & 4 & $5,46^{*}$ & $4,95^{*}$ & $6,90^{*}$ & $46,33^{*}$ & $8,59^{\star}$ & $5,92^{*}$ \\
\hline$D^{*} P$ & 12 & $2,45^{\star}$ & $3,40^{*}$ & $3,71^{*}$ & $1,03 \mathrm{~ns}$ & $2,22 \mathrm{~ns}$ & $5,19^{*}$ \\
\hline RESÍDUO & 20 & - & - & - & - & - & - \\
\hline TOTAL & 39 & - & - & - & - & - & - \\
\hline
\end{tabular}

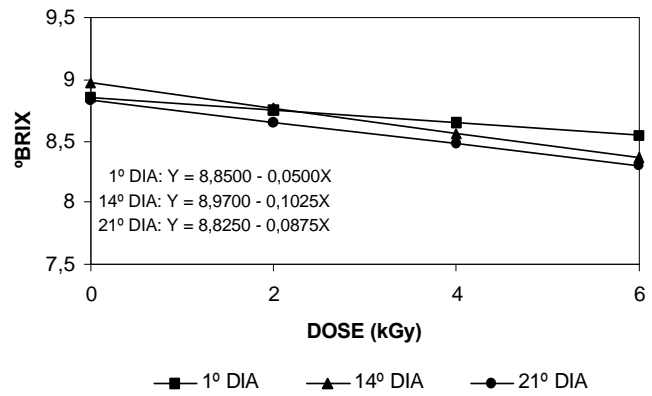

Figura 1 - Variação do teor de sólidos solúveis ('Brix) do suco de laranja em função da dose de radiação, durante o período de armazenamento.

Houve uma diminuição do teor de sólidos solúveis com o aumento da dose de radiação no $1^{\circ}, 14^{\circ}$ e $21^{\circ}$ dia de armazenamento.

Os resultados obtidos estão de acordo com Yáñez et al. (1990) e Arteaga et al. (1988), os quais trabalharam com laranjas irradiadas com doses de 0,25 a 1,50 kGy e obtiveram diminuição no teor de sólidos solúveis com o aumento da dose de radiação recebida pelas laranjas.

O oposto foi observado por Spoto (1988) em suco de laranja irradiado com várias doses. Munhoz-Burgos (1985) também observou este fato em suco de tangerina, ao passo que no suco de laranja o conteúdo de sólidos totais permaneceu estável.

A acidez titulável sofreu efeito da interação dose de radiação e período de armazenamento (TABELA 1). Verificou-se a existência de uma relação quadrática entre as doses e o teor de ácido cítrico no $1^{\circ}, 7^{\circ}$ e $28^{\circ}$ dia de armazenamento (Figura 2), enquanto que nos demais períodos não foi observada nenhuma relação.

Derivando-se as equações (Figura 2)

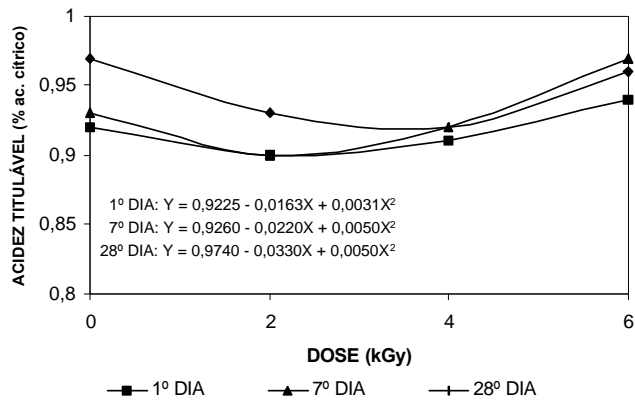

Figura 2 - Variação da acidez titulável do suco de laranja em função da dose de radiação, durante o período de armazenamento.

obtiveram-se as doses 2,63 kGy, 2,20 kGy e 3,30 kGy, responsáveis pelos teores mínimos de ácido cítrico no $1^{\circ}(0,90 \%)$, no $7^{\circ}(0,90 \%)$ e no $28^{\circ}$ dia de armazenamento $(0,92 \%)$, respectivamente .

Estes resultados coincidem com os encontrados por outros autores, os quais também verificaram uma diminuição na acidez com o aumento da dose de radiação em laranjas tratadas com doses de 0,25 a 1,50 kGy (Yáñez et al., 1990; Arteaga et al., 1988) e em suco de laranja tratado com doses de 0,25 a 2,0 kGy (Munhoz-Burgos, 1985).

Houve efeito significativo da interação dose de radiação e período de armazenamento sobre a razão SS/AT (TABELA 1). No $1^{\circ}, 7^{\circ} \mathrm{e}$ $28^{\circ}$ dia de armazenamento foi encontrada uma razão quadrática entre a dose de radiação e a relação SS/AT, enquanto que no $14^{\circ}$ e $21^{\circ}$ dia houve uma relação linear (Figura 3 ).

Derivando-se as equações (Figura 3) obtiveram-se as doses 2,10 kGy, 2,47 kGy e 2,88 kGy, responsáveis pelos teores máximos da razão SS/AT no $1^{\circ}(9,79)$, no $7^{\circ}(9,25)$ e no $28^{\circ}$ dia de armazenamento $(9,57)$, respectivamente. 
No $14^{\circ}$ e $21^{\circ}$ dia de armazenamento houve uma diminuição da razão SS/AT com o aumento da dose de radiação.

Conforme indica o TABELA 1, houve efeito significativo da dose de radiação (Figura 4) e do período de armazenamento (Figura 5) sobre $0 \mathrm{pH}$. Derivando-se a equação de regressão (Figura 4) obteve-se a dose de 2,62 kGy, responsável pelo maior valor de $\mathrm{pH}(3,70)$. Derivando-se a equação de regressão (Figura 5) obteve-se no $17^{\circ}$ dia o maior valor de $\mathrm{pH}(3,69)$.

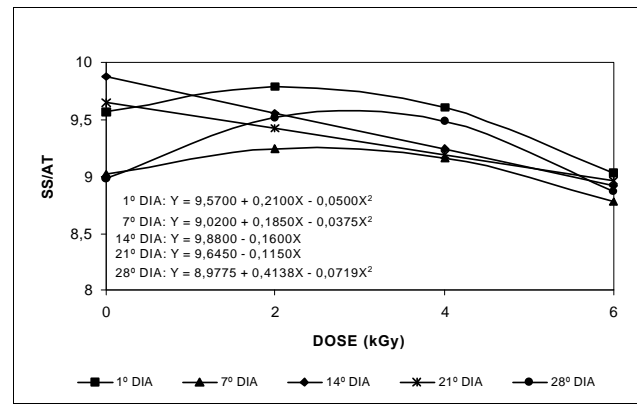

Figura 3 - Variação da razão SS/AT do suco de laranja em função da dose de radiação, durante o período de armazenamento.

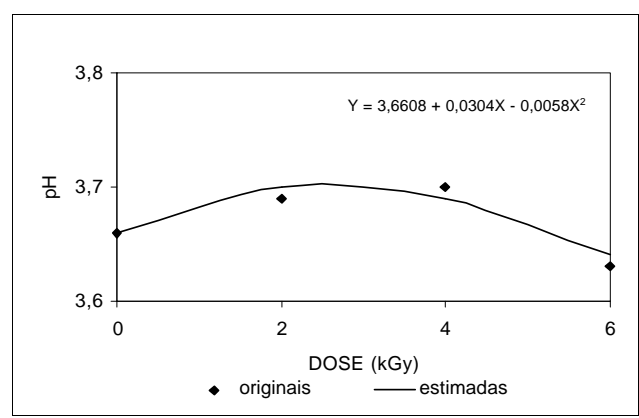

Figura 4 - Variação do pH do suco de laranja em função da dose de radiação.

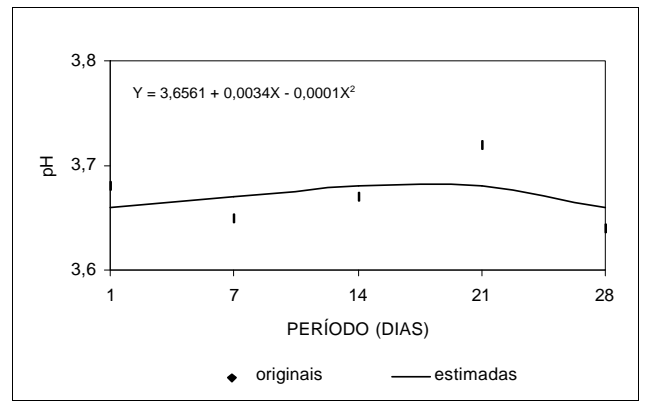

Figura 5 - Variação do pH do suco de laranja durante o período de armazenamento.
Estes resultados coincidem com os de Yáñez et al. (1990) e Arteaga et al. (1988), os quais trabalharam com laranjas irradiadas com doses de 0,25 a 1,50 kGy e obtiveram aumento no $\mathrm{pH}$ com o aumento da dose de radiação recebida pelas laranjas.

Os resultados também concordam com Maxie et al. (1969), que observaram um pH ligeiramente superior em laranjas irradiadas com doses de até 2,0 kGy.

Wilska-Jeszka \& Skorupinska (1975) observaram diminuição no $\mathrm{pH}$ do suco de tomate e dos xaropes de groselha preta e vermelha com o aumento das doses de radiação, enquanto que Kiss (1973) observou um acréscimo do pH em diversas variedades de uva com o aumento da dose de radiação. Já Nagai \& Moy (1985) não observaram diferença no $\mathrm{pH}$ de suco de pera e de nectarina irradiados.

De modo geral, pode-se inferir que, embora havendo diferença estatística, as variações encontradas neste trabalho para teor de sólidos solúveis, acidez total, razão SS/AT e $\mathrm{pH}$ foram mínimas e pouco alteraram as características químicas do suco de laranja irradiado. Resultados semelhantes foram obtidos por Spoto (1988), a qual, trabalhando com suco de laranja irradiado com doses de 2,5; 5,0 e 7,5 kGy, concluiu que as diferenças encontradas para teor de sólidos solúveis, acidez total e $\mathrm{pH}$, embora significativas, foram insuficientes para alterar as características químicas e sensoriais do suco irradiado.

Conforme indica o TABELA 1, houve efeito significativo da dose de radiação (Figura 6) e do período de armazenamento (Figura 7) sobre o teor de ácido ascórbico. Os resultados mostram que houve uma diminuição do teor de ácido ascórbico em função do aumento da dose de radiação (Figura 6) e do período de armazenamento (Figura 7).

A redução no conteúdo de ácido ascórbico pela irradiação tem sido observada em frutas cítricas (Thomas, 1986; Arteaga et al., 1988; Yáñez et al., 1990), suco de tomate e xaropes de groselha preta e vermelha (WilskaJeszka \& Skorupinska, 1975) e suco de laranja (Hussain \& Maxie, 1974).

Em estudo realizado por Spoto (1988), tanto a dose quanto a temperatura de irradiação provocaram apenas ligeira diminuição na porcentagem de ácido ascórbico do suco de laranja. Esta porcentagem foi mais drasticamente diminuída pela temperatura e pelo período de armazenamento. 


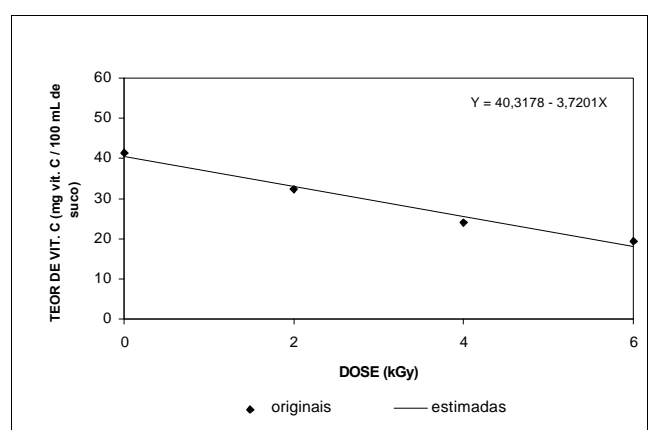

Figura 6 - Variação do teor de ácido ascórbico do suco de laranja em função da dose de radiação.

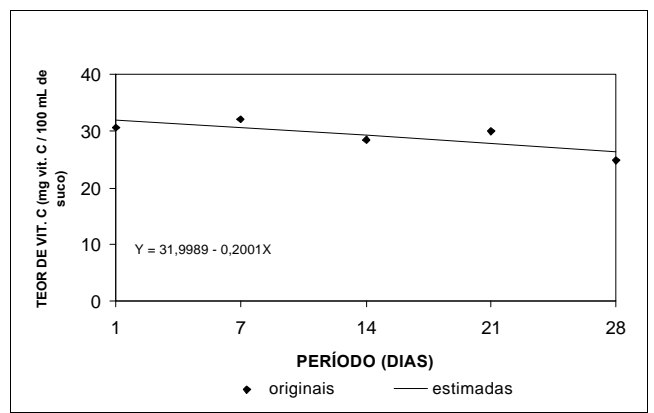

Figura 7 - Variação do teor de ácido ascórbico do suco de laranja durante o período de armazenamento.

Os resultados das análises do conteúdo de vitamina $C$ do suco logo após sua irradiação, comparados com o controle, mostram que a perda desta vitamina pela irradiação foi de $17 \%$ para a dose de $2,0 \mathrm{kGy}$, de $45 \%$ para a dose de 4,0 kGy e de $55 \%$ para a dose de 6,0 kGy. Resultados semelhantes de perda da vitamina com a aplicação da dose de 2,0 kGy foram observados por Beyers \& Thomas (1979) em mangas, e por Arteaga et al. (1988) e Yáñez et al. (1990) em laranjas. No entanto, Maxie \& Sommer (1968) observaram apenas 3,6\% de perda da vitamina em laranjas irradiadas com esta dose.

Maxie et al. (1964) não observaram perdas significativas de ácido ascórbico em laranjas irradiadas e armazenadas a $0^{\circ} \mathrm{C}$ por 100 dias. No entanto, verificaram redução no conteúdo de ácido ascórbico em limões irradiados e armazenados a $15^{\circ} \mathrm{C}$ por um mês.

Houve efeito significativo da interação dose de radiação e período de armazenamento sobre a contagem microbiológica total, em $\mathrm{UFC} / \mathrm{mL}$ (TABELA 1). No $1^{\circ}$ dia houve uma relação linear, enquanto que no $7^{\circ}, 14^{\circ}, 21^{\circ}$ e $28^{\circ}$ dia de armazenamento houve uma relação quadrática entre a dose de radiação e a população microbiana (Figura 8).

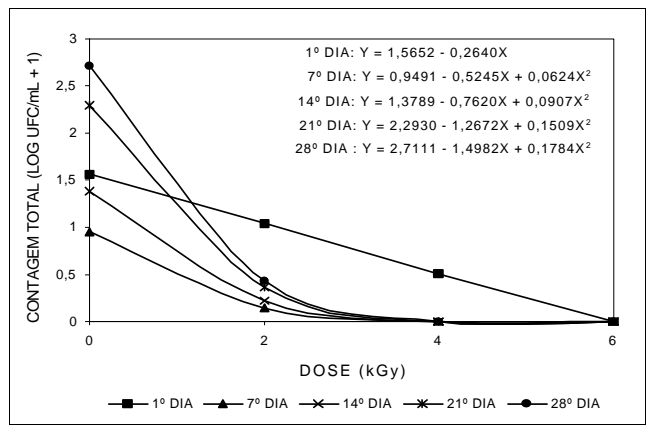

Figura 8 - Variação da população microbiana (Log $\mathrm{UFC} / \mathrm{mL}+1$ ) do suco de laranja em função da dose de radiação, durante o período de armazenamento.

Pela Figura 8, observa-se que houve uma diminuição da população microbiana com o aumento da dose de radiação. No primeiro dia de armazenamento não foi detectada nenhuma unidade formadora de colônia por $\mathrm{mL}$ do suco de laranja com a aplicação da dose de 6,0 kGy. Nos demais períodos de armazenamento, já com a utilização da dose de 4,0 kGy não foi constatada nenhuma unidade formadora de colônia por $\mathrm{mL}$ do suco de laranja.

Os resultados de diminuição da população microbiana com o aumento da dose de radiação estão de acordo com Domarco et al. (1996), os quais obtiveram inibição na proliferação da levedura Saccharomyces cerevisiae em suco de laranja irradiado com doses de 5,0 e 7,5 kGy e armazenados por 90 dias a $25^{\circ} \mathrm{C}$. Estes resultados também concordam com Alcarde \& Walder (1997), que verificaram redução na população de Saccharomyces cerevisiae em mosto de mel de cana-de-açúcar com o aumento da dose de radiação.

\section{CONCLUSÕES}

O conteúdo de vitamina $C$ do suco de laranja foi afetado pela dose de radiação e pelo período de armazenamento porque ambos provocaram redução no conteúdo da vitamina no suco.

A irradiação gama pode ser um processo eficaz na conservação de suco de laranja porque reduziu a população microbiana do suco armazenado por até 28 dias. 


\section{REFERÊNCIAS BIBLIOGRÁFICAS}

ALCARDE, A.R.; WALDER, J.M.M. Efeito da radiação gama na sobrevivência da levedura Saccharomyces cerevisiae (cepa M-300-A) em mosto de mel de cana-de-açúcar. Scientia Agricola, v.54, n.3, p.203-208, 1997.

ARTEAGA, A.G.; YÁÑEZ, M.G.; MIRANDA, J.F.; PARADOA, A.; SAMPERE, E.; SERRANO, G.; CASTILLO, E. Estabilidad del contenido de vitamina $\mathrm{C}$ e algunos indicadores químicos en naranjas sometidas a diferentes dosis de radiaciones gamma. Revista Alimentaria, v.198, p.45-46, 1988.

BEYERS, M.; THOMAS, A. Irradiation of subtropical fruits : changes in certain nutrients presents in mangoes, papayas and litchis during caning, freezing and gamma irradiation. Journal of Agriculture and Food Chemistry, v.27, n.1, p.48-51, 1979.

DOMARCO, R.E.; SPOTO, M.H.F.; WALDER, J.M.M.; BLUMER, L.; MATRAIA, C. Efeitos do tratamento combinado de irradiação gama e refrigeração no crescimento de levedura (Saccharomyces cerevisiae Hansen) em suco de laranja. Scientia Agricola, v.53, n.1, p.14-19, 1996.

HUSSAIN, N.H.; MAXIE, E.C. Effect of gamma rays on shelf life and quality on orange juice. International Biodeterioration Bulletin, v.10, n.3, p.81-86, 1974.

JACOBS, M.B. The chemical analysis of foods and food products. New York: Van Nostrand, 1958. 979p.

JOSEPHSON, E.S. Nutritional aspects os food irradiation: an overview. Journal of Food Processing and Preservation, v.2, p.299-313, 1978.

KISS, I. Radiation preservation of fruit juice semiconcentrates prepared by cryoconcentration. Acta Alimentaria, v.2, n.3, p.275-283, 1973.

MAXIE, E.C.; SOMMER, N.F. Changes in some chemicals constituents in irradiates fruits and vegetables. In: INTERNATIONAL ATOMIC ENERGY AGENCY. Preservation of fruits and vegetables by irradiation. Vienna: IAEA, 1968. p.36-53.

MAXIE, E.C.; EAKS, I.L.; SOMMER, N.F. Some physiological effects of gamma irradiation on lemon fruit. Radiation Botany, v.4, p.405-411, 1964.
MAXIE, E.C.; SOMMER, N.F.; EAKS, I.L. Effect of gamma radiation on citrus fruits. In: INTERNATIONAL CITRUS SYMPOSIUM, California, 1969. Proceedings. Vienna: IAEA, 1969. p.1375-1387.

MUNHOZ-BURGOS, R.A. Uso de la radiación gama para extender el tiempo de conservatión del jugos de algunas frutas exóticas. In: SEMINÁRIO SOBRE IRRADIACIÓN DE ALIMENTOS PARA PAÍSES DA AMÉRICA LATINA, 1., Quito, 1985. Actas. Vienna: IAEA, 1985. p.1-25.

NAGAI, N.Y.; MOY, J.H. Quality of gamma irradiated California and Valência oranges. Journal of Food Science, v.50, n.1, p.215-219, 1985.

SAS Institute. SAS user's guide: statistics. Cary: Statistical Analysis System Institute, 1990. 584p.

SPOTO, M.H.F. Radiação gama na conservação do suco concentrado de laranja: características físicas, químicas e sensoriais. Piracicaba, 1988. 88p. Dissertação (Mestrado) - Escola Superior de Agricultura "Luiz de Queiroz", Universidade de São Paulo.

SWANSON, K.M.J.; BUSTA, F.F.; PETERSON, E.H. Colony count methods. In: VANDERZANT, C.; SPLITTSTROESSER, D.F. (Ed.) Compendium of methods for the microbiological examination of foods. 3ed. Washington: American Public Health Association, 1992. 1219p. cap.4, p.75-95.

THOMAS, P. Radiation preservation of feed of plant origin: subtropical fruits: citrus, grapes and avocados. Critical Reviews in Food Science and Nutrition, v.24, n.1, p.53-89, 1986.

WIENDL, F.M. A salubridade dos alimentos irradiados. Boletim da Sociedade Brasileira de Ciência e Tecnologia de Alimentos, v.18, n.1, p.48-56, 1984.

WILSKA-JESZKA, J.; SKORUPINSKA, A. Evaluation of radurized and pasteurized fruits juices during 12 months of storage. Seszyty Naukowe Politechniki LodzkiejChemia Spozywcza, v.23, p.243-259, 1975.

YÁÑEZ, M.G.; ARTEAGA, A.G.; MIRANDA, J.F.; PARADOA, A.; SAMPERE, E.; CASTILLO, E.; SERRANO, G. Nota: Estabilidad del contenido de vitamina $\mathrm{C}$ en toronjas sometidas a diferentes dosis de radiaciones gamma. Revista de Agroquímica e Tecnologia de Alimentos, v.30, n.3, p.409-416, 1990.

Recebido para publicação em 19.01.99

Aceito para publicação em 02.08.99 